\title{
[gw22-e1024] GENETICALLY ENGINEERED MESENCHYMAL STEM CELLS TO CREATE CARDIAC PACEMAKER CELLS
}

Ya-feng Zhou, Xiang-jun Yang, Hong-xia Li, Lian-hua Han, Wen-ping Jiang Department of Cardiology, The First Affiliated Hospital of Soochow University, Suzhou City, Jiangsu Province, China

10.1136/heartjnl-2011-300867.255

Objective The study was to test proof-of-principle if genetically engineered mesenchymal stem cells (MSCs) transfected with HCN2 genes can be modified to be cardiac pacemaker cells.

Methods (1) MSCs of rabbit were isolated from the posterior iliac crest of rabbit and were used from passages 2 to 4 . (2) The self-inactivating HIV1-based lentiviral vector (LentiV) was used as transgene delivery, which was constructed with plasmid hHCN2/pcDNA3. (3) Total RNA was extracted from control MSCs and those transfected with hHCN2, and RT-PCR was performed. (4) Membrane proteins were extracted from control MSCs and those transfected with hHCN2. Western blot analysis was performed. (5) Whole-cell patch clamp was used to study membrane currents.

Results (1) In addition to expressing characteristic hHCN2 protein, mHCN2-transfected hMSCs also express an anticipated high level of hHCN2 gene by RT-PCR and Western blot analysis. (2) $\mathrm{I}_{\mathrm{f}}$ was elicited using hyperpolarising steps in $10-\mathrm{mV}$ increments from -40 to $-140 \mathrm{mV}$, and it was significantly inhibited by $4 \mathrm{mM}$ cesium chloride. (3) The coculture beating rate of cardiac myocytes was $87 \pm 11 \mathrm{bpm}$ when MSCs were transfected with control plasmid (expressing only GFP) and $149 \pm 14 \mathrm{bpm}$ when MSCs were expressing both GFP+hHCN2 $(\mathrm{p}<0.05)$.

Conclusions The MSC expressing hHCN2 is a demonstration of feasibility of preparing MSC-based biological pacemaker cells. MSCs transfected with hHCN2 genes by LentiV are 
capable of actively pacing ventricular cardiac myocytes and can be modified to be cardiac pacemaker cells. 